\title{
Zika Virus Infection in Pregnant Women in Rio de Janeiro
}

\author{
P. Brasil, J.P. Pereira Jr., M.E. Moreira, R.M. Ribeiro Nogueira, L. Damasceno, M. Wakimoto, \\ R.S. Rabello, S.G. Valderramos, U.-A. Halai, T.S. Salles, A.A. Zin, D. Horovitz, P. Daltro, M. \\ Boechat, C. Raja Gabaglia, P. Carvalho de Sequeira, J.H. Pilotto, R. Medialdea-Carrera, D. \\ Cotrim da Cunha, L.M. Abreu de Carvalho, M. Pone, A. Machado Siqueira, G.A. Calvet, A.E. \\ Rodrigues Baião, E.S. Neves, P.R. Nassar de Carvalho, R.H. Hasue, P.B. Marschik, C. \\ Einspieler, C. Janzen, J.D. Cherry, A.M. Bispo de Filippis, and K. Nielsen-Saines
}

\begin{abstract}
BACKGROUND—Zika virus (ZIKV) has been linked to central nervous system malformations in fetuses. To characterize the spectrum of ZIKV disease in pregnant women and infants, we followed patients in Rio de Janeiro to describe clinical manifestations in mothers and repercussions of acute ZIKV infection in infants.
\end{abstract}

METHODS-We enrolled pregnant women in whom a rash had developed within the previous 5 days and tested blood and urine specimens for ZIKV by reverse-transcriptase-polymerase-chainreaction assays. We followed women prospectively to obtain data on pregnancy and infant outcomes.

RESULTS-A total of 345 women were enrolled from September 2015 through May 2016; of these, 182 women (53\%) tested positive for ZIKV in blood, urine, or both. The timing of acute ZIKV infection ranged from 6 to 39 weeks of gestation. Predominant maternal clinical features included a pruritic descending macular or maculopapular rash, arthralgias, conjunctival injection, and headache; $27 \%$ had fever (short-term and low-grade). By July 2016, a total of 134 ZIKVaffected pregnancies and 73 ZIKV-unaffected pregnancies had reached completion, with outcomes known for $125 \mathrm{ZIKV}$-affected and $61 \mathrm{ZIKV-unaffected} \mathrm{pregnancies.} \mathrm{Infection} \mathrm{with} \mathrm{chikungunya}$ virus was identified in $42 \%$ of women without ZIKV infection versus $3 \%$ of women with ZIKV infection $(\mathrm{P}<0.001)$. Rates of fetal death were $7 \%$ in both groups; overall adverse outcomes were $46 \%$ among offspring of ZIKV-positive women versus $11.5 \%$ among offspring of ZIKV-negative women $(\mathrm{P}<0.001)$. Among 117 live infants born to $116 \mathrm{ZIKV}$-positive women, $42 \%$ were found to have grossly abnormal clinical or brain imaging findings or both, including 4 infants with microcephaly. Adverse outcomes were noted regardless of the trimester during which the women were infected with ZIKV (55\% of pregnancies had adverse outcomes after maternal infection in the first trimester, 52\% after infection in the second trimester, and 29\% after infection in the third trimester).

Address reprint requests to Dr. Brasil at Laboratorio de Doenças Febris Agudas, INI, Fiocruz, 4365 Av Brasil, Rio de Janeiro-RJ 21040-900, or at patricia.brasil33@gmail.com, or to Dr. Nielsen-Saines at the Division of Pediatric Infectious Diseases, David Geffen School of Medicine at UCLA, MDCC 22-442, 10833 LeConte Ave., Los Angeles, CA 90095, or at knielsen@ mednet.ucla.edu.

The authors' full names, academic degrees, and affiliations are listed in the Appendix.

No potential conflict of interest relevant to this article was reported.

Disclosure forms provided by the authors are available with the full text of this article at NEJM.org. 
CONCLUSIONS-Despite mild clinical symptoms in the mother, ZIKV infection during pregnancy is deleterious to the fetus and is associated with fetal death, fetal growth restriction, and a spectrum of central nervous system abnormalities. (Funded by Ministério da Saúde do Brasil and others.)

We have been conducting active surveillance for dengue infection in the general population of Rio de Janeiro since 2007. In 2012, we established a prospective cohort for dengue surveillance in mother-infant pairs within the Manguinhos Rio de Janeiro area. In 2015, we noted an increase in cases of a dengue-like illness that was characterized by a descending rash, generally without fever; this increase coincided with a surge in the number of cases of illness characterized by a pruriginous rash in northeastern Brazil. ${ }^{1}$ In early 2015, most cases were originally reported to surveillance systems as dengue; however, Zika virus (ZIKV) was eventually identified. ${ }^{2-8}$ To identify ZIKV cases in our population, we modified our pregnancy cohort study and enrolled women who presented with a rash at any week of gestation. This report is a follow-up to a previously published preliminary report, available with the full text of this article at NEJM.org.

\section{METHODS}

\section{STUDY POPULATION}

In this cohort study, pregnant women at any week of gestation who presented to the acute febrile illness clinic at the Oswaldo Cruz Foundation with a rash that had developed within the previous 5 days were offered enrollment and were included in the study after they had provided written informed consent. After the women were enrolled, detailed demographic, medical, and prenatal history information, as well as clinical findings, were entered into case-report forms. Laboratory data on rubella, cytomegalovirus, and Venereal Disease Research Laboratory (VDRL) serologic testing were abstracted from prenatal medical records and entered in case-report forms. Serum and urine specimens were obtained at study entry. Weekly follow-up was conducted by telephone, and a second visit was scheduled within 30 days after enrollment for clinical and laboratory follow-up. Women were referred for fetal ultrasonography before 20 weeks of gestation, between 20 and 30 weeks of gestation, and after 30 weeks of gestation. No women had had a diagnosis of fetal malformations in the current pregnancy before enrollment. The study population was generally healthy; women reported no coexisting conditions or medication use. Infants born to ZIKV-positive mothers are being followed prospectively.

\section{STUDY OVERSIGHT}

The study protocol was approved by the institutional review boards at Fundação Oswaldo Cruz (Fiocruz) and the University of California, Los Angeles. The authors vouch for the accuracy and completeness of the data and the analyses and for the fidelity of the study to the protocol.

\section{LABORATORY TESTING}

Real-time reverse-transcriptase-polymerase-chain-reaction (RT-PCR) assays for ZIKV were performed with the QuantiTect Probe RT-PCR kit (Qiagen), as described previously, ${ }^{9}$ with 
the same primers and cycle times, at the Fiocruz Flavivirus Laboratory; assays were performed on blood specimens, urine specimens, or both that were obtained at the entry visit. The Fiocruz Flavivirus Laboratory is a reference laboratory for flavivirus infections in the region. Serologic testing for IgG antibodies to dengue (Abcam) and IgM antibodies to chikungunya (Euroimmun) were performed on serum specimens obtained at the entry visit. Patient specimens were also tested by RT-PCR for dengue ${ }^{10}$ and chikungunya. ${ }^{9}$ Patients were tested for parvovirus B19 and cytomegalovirus by PCR (TaqMan RT PCR, Applied Biosystems) and by serologic testing during regular prenatal care. PCR assays for human immunodeficiency virus (HIV) DNA (Abbott RT HIV Viral Load) were performed on all patient specimens. Screening tests for syphilis were performed during prenatal care with the use of VDRL assays, with treponemal assays (Alere Determine Syphilis) used as confirmatory tests.

\section{FETAL ULTRASONOGRAPHY}

All abdominal scanning was performed with a 4-to-8-mHz probe (Voluson 730 Expert/ Voluson E6, GE) by perinatologists who were certified by the Brazilian College of Radiology and the Brazilian Federation of Societies of Gynecology and Obstetrics (Febrasgo). The variables that were measured are listed in the Supplementary Appendix, available at NEJM.org. For Doppler studies, the pulsatility index of the umbilical artery and of the middle cerebral artery were used. ${ }^{11}$ Abnormalities such as cerebral calcifications and microcephaly were noted. Measured fetal ultrasonographic variables were plotted by gestational age according to the nomograms published on www.perinatology.com. Fetal growth restriction was defined as fetal weight estimated according to the Hadlock formula that was below the 10th percentile. ${ }^{12}$ Microcephaly in fetal imaging was defined as fetal head measurements (e.g., head circumference) that were two standard deviations below the mean expected at a particular gestational age or below the 3 rd percentile. ${ }^{13}$

\section{INFANT CLINICAL ASSESSMENTS}

Anthropometric measures at birth (weight, length, and head circumference) were obtained in all live-born infants. History taking and physical examinations were performed for all infants by pediatric specialists. ZIKV-exposed infants were evaluated by a multidisciplinary team that included neonatologists, neurologists, infectious disease specialists, geneticists, ophthalmologists, and physical therapists. All abnormal diagnoses were reviewed by a panel of specialists for confirmation. Microcephaly was defined as a head-circumference $\mathrm{z}$ score of less than -2 (moderate) or less than -3 (severe). Small-for-gestational-age infants were defined as infants with body-weight $\mathrm{z}$ scores of less than -1.28 at birth. ${ }^{14}$

\section{INFANT IMAGING STUDIES}

Brain imaging studies were offered for infants born to mothers who had positive PCR results for ZIKV. Transfontanel ultrasonography, computed tomography (CT) of the head with and without contrast, and magnetic resonance imaging (MRI; AERA Siemens, 1.5 tesla) with or without contrast were performed according to the standard of care. If abnormalities were suspected on transfontanel ultrasonography, patients were referred for CT or MRI brain imaging. All infant assessments were conducted with knowledge of ZIKV infection status. 


\section{STATISTICAL ANALYSIS}

We compared the demographic and clinical variables of pregnant women who were positive for ZIKV on PCR with those who were negative for ZIKV on PCR, using Fisher's exact test (two-sided); $P$ values of 0.05 or less were considered to indicate statistical significance. We performed similar analyses comparing pregnancy and infant outcomes in ZIKV-infected and ZIKV-uninfected mother-infant pairs. For comparison of medians, an independent-samples median test was used. Comparisons of negative pregnancy outcomes according to maternal trimester of infection between ZIKV-infected and ZIKV-uninfected mother-infant pairs were compared with the use of McNemar's test.

\section{RESULTS}

\section{CHARACTERISTICS OF THE PARTICIPANTS}

During the period from September 2015 through May 2016, we enrolled 345 pregnant women and tested blood specimens, urine specimens, or both for ZIKV by qualitative RTPCR. Of these 345 women, 182 (53\%) had positive results for ZIKV on PCR in blood, urine, or both. The current report focuses on $134 \mathrm{ZIKV}$-positive women and $73 \mathrm{ZIKV}$-negative women who were expected to deliver by July 31, 2016 (Fig. 1). Among the 134 ZIKVpositive women, quantitative ZIKV PCR results were available for 130 (4 PCR assays were performed at outside facilities). Among women with quantitative PCR results, 85 had positive PCR results in serum specimens, 76 had positive PCR results in urine, and 31 had positive PCR results in both specimens; 45 women had positive results in urine only and 54 had positive results in blood specimens only (median number of PCR cycles for serum specimens, 32.0; inter-quartile range, 30.0 to 34.0 ; range, 24.2 to 37.0 ; and median number of PCR cycles for urine specimens, 30.0; interquartile range, 27.0 to 33.0; range, 22.0 to 37.0). Demographic and clinical characteristics are described in Table 1. Among ZIKVpositive women, $38 \%$ reported similar illnesses in other family members, and only $14 \%$ reported that their partner had been ill. ZIKV infection was present in women of all socioeconomic strata. More than half the women presented with acute infection in the second trimester of pregnancy. ZIKV-negative women were more likely to have used insect repellent than ZIKV-positive women ( $83 \%$ vs. $60 \%, \mathrm{P}=0.006$ ), but otherwise there were no significant differences in demographic characteristics or medical history between the two groups. ZIKV-positive women resided across multiple neighborhoods and municipalities within the larger metropolitan Rio de Janeiro area (Fig. S1 in the Supplementary Appendix).

\section{CLINICAL PRESENTATION OF THE MOTHERS}

All pregnant women had rash as part of their clinical presentation, since rash was an inclusion criterion. A descending macular or maculopapular rash was the most common type of exanthem noted in ZIKV-positive women (Fig. S5 in the Supplementary Appendix). The maculopapular rash was seen far more frequently in ZIKV-positive women than in ZIKVnegative women $(\mathrm{P}=0.02)$. The other prevalent finding was pruritus, which was seen in $90 \%$ of ZIKV-positive women in our study. The next most common finding was arthralgia, which was reported in $62 \%$ of ZIKV-positive women and in 70\% of ZIKV-negative women $(\mathrm{P}=0.29)$. Conjunctival injection was present in $58 \%$ of $\mathrm{ZIKV}$-positive women and in a smaller percentage $(40 \%)$ of ZIKV-negative women $(P=0.03)$. Fatigue or malaise was 
described in 52\% of ZIKV-positive women versus 75\% of ZIKV-negative women (P = $0.002)$, and myalgia was described in $41 \%$ versus $62 \%(P=0.005)$; the higher rates among $\mathrm{ZIKV}$-negative women were probably due to the diagnosis of underlying chikungunya virus in some ZIKV-negative women. Lymphadenopathy (isolated or generalized) was present in both groups (in 38\% of ZIKV-positive women and $27 \%$ of ZIKV-negative women, $\mathrm{P}=0.12$ ). Fever was not a highly prominent finding, occurring in less than a third of the women with acute ZIKV infection but in 58\% of ZIKV-negative women ( $\mathrm{P}<0.001)$. When fever was present, it was generally short-term and low grade $\left(37.5\right.$ to $\left.38.0^{\circ} \mathrm{C}\right)$. Nausea or vomiting was reported in $31 \%$ of ZIKV-positive women and was more common (occurring in 44\%) among ZIKV-negative women ( $\mathrm{P}=0.07)$.

\section{OUTCOMES OF PREGNANCIES}

Among 134 women who had positive results for ZIKV on PCR testing, information on confirmed outcomes of pregnancy was available for 125, with 117 live births in 116 pregnancies (one set of twins) between January 1 and July 31, 2016. There were 9 cases of fetal death (Fig. 1 and Table 2, and Table S2 in the Supplementary Appendix): 5 miscarriages in the first trimester of pregnancy, 2 miscarriages in the second trimester, and 2 stillbirths in the third trimester. Among 73 ZIKV-negative women, 61 had known outcomes, including 4 cases of fetal death and 57 live births. In the ZIKV-positive group, 93\% of the women remained in the study, whereas retention in the ZIKV-negative group was $84 \%$ ( $\mathrm{P}=$ $0.03)$.

Three of seven negative pregnancy outcomes in the ZIKV-negative group - two of the four fetal losses and one small-for-gestational-age infant - occurred in women with chikungunya virus. One of the first-trimester miscarriages in the ZIKV-positive cohort occurred in a woman who was coinfected with ZIKV and chikungunya virus. Three ZIKVinfected patients (2.8\%) were coinfected with chikungunya virus; however, chikungunya infection was more prevalent in the ZIKV-negative group (42\%, P<0.001) (Table 2). No patients in either group were found to have active dengue infection on PCR; however, prior dengue infection was very common, with $88 \%$ in the ZIKV-positive group and $86 \%$ in the ZIKV-negative group having IgG antibodies to dengue. One woman in each group had a positive treponemal syphilis test; those pregnancies did not have adverse outcomes. No women had evidence of active cytomegalovirus infection by either PCR or IgM detection. Among ZIKV-negative women, 29 other infections were identified, including 23 cases of chikungunya, 4 cases of parvovirus B19, and 1 case of syphilis. None of the women were found to have HIV by RT-PCR testing.

Among 125 pregnancies in ZIKV-positive women, 58 adverse pregnancy outcomes were noted (46.4\%); in contrast, 7 of the 61 pregnancies (11.5\%) in the ZIKV-negative cohort resulted in adverse outcomes $(\mathrm{P}<0.001)$. Despite the high rate of adverse outcomes in our control group of pregnant women with other infectious illnesses, the findings in the ZIKVpositive group were far more striking. Adverse pregnancy outcomes by the timing of maternal ZIKV infection are shown in Figure 2. The timing of maternal ZIKV infection ranged from 6 to 39 weeks of gestation. Adverse outcomes after ZIKV infection occurred regardless of the timing of maternal infection; adverse outcomes occurred in 55\% of 
pregnancies in which the mother was infected in the first trimester (11 of $20 \mathrm{ZIKV}$-infected pregnancies), in 52\% of those in which the mother was infected in the second trimester (37 of $71 \mathrm{ZIKV}$-infected pregnancies), and in $29 \%$ of those in which the mother was infected in the last trimester of pregnancy (10 of $34 \mathrm{ZIKV}$-infected pregnancies). Among ZIKV-infected pregnancies, there were 5 miscarriages ( $25 \%$ of the 20 pregnancies with first-trimester infection), 2 fetal losses (3\% of the 71 pregnancies with second-trimester infection); and 2 stillbirths (6\% of the 34 pregnancies with third-trimester infection). Among 117 live births in the ZIKV-positive cohort, 49 infants (42\%) were found to have abnormalities on clinical examination, imaging, or both; in contrast, among 57 live births in the ZIKV-negative cohort, 3 infants $(5 \%)$ had such abnormalities $(\mathrm{P}<0.001)$.

Three infants with abnormalities who were born to ZIKV-negative women were small for gestational age; one was born to a woman who was found to have chikungunya virus infection. Because of the large number of chikungunya infections in the control group, adverse pregnancy outcomes were also frequent in this group. There was no significant difference in the rate of fetal loss between ZIKV-positive mothers and ZIKV-negative mothers $(7.2 \%$ and $6.6 \%$, respectively; $\mathrm{P}=1.0)$. Obstetrical complications were very high in both groups: $39 \%$ in ZIKV-positive women and 35\% in ZIKV-negative women $(\mathrm{P}=0.62)$. ZIKV-positive women, however, were nearly 10 times as likely as ZIKV-negative women to have emergency cesarean sections performed owing to fetal distress $(23.5 \%$ vs. $2.5 \%, \mathrm{P}=$ 0.003) (Table 2). Infants born to ZIKV-positive mothers were also nearly 4 times as likely to need critical care assistance immediately after birth (a finding that is reflective of fetal distress) as infants who had not been exposed to ZIKV ( $21 \%$ vs. $6 \%, \mathrm{P}=0.01)$.

A total of 153 ultrasound studies were performed in 59 women who were found to be positive for ZIKV during pregnancy; the remaining 75 ZIKV-positive women declined imaging studies either because the obstetrical facility was too far away or because of fear of possible fetal abnormalities related to ZIKV infection. Detailed ultrasonographic findings are described in Table S1 in the Supplementary Appendix. ZIKV-negative women underwent fetal ultrasonography as part of regular prenatal care. All the women in the cohort received prenatal care. Nine women with ZIKV infection (6.7\%) were lost to follow-up (i.e., did not seek prenatal care at our obstetrical facility and were unable to be reached for further evaluation despite multiple attempts; pregnancy outcomes are unknown). Included among these 9 women was 1 woman whose fetus had severe abnormalities on fetal ultrasonography. In the ZIKV-negative cohort, 12 women (16\%) were lost to follow-up, mainly because of a change in their contact information. ZIKV-negative women did not deliver at our obstetrical facility. In addition, women were less likely to continue follow-up in the study if ZIKV infection was ruled out.

\section{INFANT OUTCOMES}

Infants who were small for gestational age, as a potential consequence of fetal growth restriction or poor placental perfusion, constituted $9 \%$ of ZIKV-exposed babies and $5.3 \%$ of babies in the control group $(\mathrm{P}=0.06)$. Four infants in the $\mathrm{ZIKV}$-positive group $(3.4 \%)$ were noted to have microcephaly at birth; two were small-for-gestational-age infants with proportionate microcephaly (i.e., the head size is small but is proportional to the weight and 
length of the infant), and two had disproportionate microcephaly (i.e., the head size is small relative to the weight and length of the infant). None of the infants in the control group had microcephaly. Fetal growth variables for fetuses of ZIKV-positive women who had ultrasonography performed during pregnancy are shown in Figure 3, and anthropometric measurements, including birth weight and head circumference, of live-born infants in both groups are shown in Figure 4 and Table 3.

A total of 49 of the 117 live-born infants (42\%) who had been exposed to ZIKV in utero had abnormal findings in the first month of life (Table S2 in the Supplementary Appendix). Almost all the abnormalities affected the central nervous system (CNS). Microcephaly was observed in infants whose mothers were infected in weeks $8,12,30$, and 38 of gestation. Disproportionate microcephaly was seen only in infants infected in the first trimester of pregnancy; 2 infants infected in later trimesters had proportionate microcephaly and were small for gestational age. One of the infants with disproportionate microcephaly was also small for gestational age. Cerebral calcifications, cerebral atrophy, ventricular enlargement, and hypoplasia of cerebral structures were seen in multiple infants, with cerebral calcifications seen in infants infected as late as 34 weeks of gestation. Parenchymal brain hemorrhages were seen in some infants, including one whose mother was infected shortly before delivery at 39 weeks of gestation. A total of 31 of the 49 infants (63\%) had grossly abnormal results on neurologic examinations; hypertonicity, clonus, hyperreflexia, abnormal movements, spasticity, contractures, and seizures were identified. Abnormal funduscopic examinations and abnormal hearing assessments were also noted.

Other features that were identified included persistence of the cortical thumb sign, with maintained clenched fists beyond 3 months of age, which reflects CNS disease; foveas in the knees or elbows due to limb contractures in utero; and redundant scalp skin in infants with normal head circumference. MRIs have not been performed in all infants to date.

Conversely, a number of infants with normal clinical assessments in early infancy had abnormal nonspecific MRI findings; a common description was "excessive hypersignaling in $\mathrm{T}_{2}$ in the white matter, diffuse in the peritrigonal posterior areas and less evident in the frontal parietal white matter with hyposignaling in the diffusion sequence." These findings are abnormal and may reflect cortical tract dysfunction; nevertheless, close follow-up will be needed to ascertain the degree of CNS involvement.

Two infants were born large for gestational age owing to maternal gestational diabetes; one of these infants, born to a mother who had been infected at 15 weeks of pregnancy, was found to have congenital heart disease, which probably was associated with maternal gestational diabetes, although we cannot exclude the possibility of a potential association with ZIKV infection. Follow-up of ZIKV-exposed infants is ongoing.

\section{DISCUSSION}

ZIKV is a flavivirus that was recently introduced into Brazil. Its rapid expansion into a population that is probably fully susceptible is due to the effectiveness of its vector, the Aedes aegypti mosquito. Diagnosis of ZIKV infection in Brazil has been complicated by the cross-reactivity among flavivirus antibodies and by the fact that dengue has been endemic in 
Brazil for more than 30 years. Serosurveillance studies have found evidence of dengue antibodies in more than $90 \%$ of the population of Recife. ${ }^{15}$ In our cohort, dengue IgG antibodies were present in $88 \%$ of the women, but no patients were found to have acute dengue infection. The diagnosis of ZIKV infection in Brazil relies on identification of the virus through RT-PCR during the acute period of infection. The virus is detectable in blood during the period of acute viremia and initial symptoms and subsequently is shed in the urine, generally for 3 to 14 days. ${ }^{16}$ Because RT-PCR assays for ZIKV are generally not available, most cases of ZIKV infection in Brazil are diagnosed clinically, without laboratory confirmation. In our study, 134 women who were positive for ZIKV had acute infection with virus that was detected in blood, urine, or both.

Nevertheless, the starting point in our cohort study was an illness with rash in a pregnant woman who presented to our clinic. As more women presented with a similar illness, we dichotomized the illnesses into two groups on the basis of the results of PCR testing for Zika virus infection. However, a number of clinical categories relating to the illnesses clearly indicate that the illnesses in the Zika-negative women differed from those in the Zikapositive women. Zika-negative women were more likely to have nausea, vomiting or anorexia, fatigue or malaise, myalgia, respiratory symptoms, and fever than were Zikapositive subjects. Also the exanthems generally differed between the two groups. As compared with women who tested negative for acute ZIKV infection, women who tested positive for the virus had distinctive clinical features that included conjunctival injection and a maculopapular pruritic rash. The presence of these clinical features should raise suspicion for ZIKV infection. Low-grade fever was observed in $27 \%$ of the women; therefore a case definition that is based on the presence of fever would miss more than $70 \%$ of cases. ${ }^{17}$

The potential cause of illness in the ZIKV-uninfected women is known in some of the cases. Rio de Janeiro saw a rise in the number of chikungunya virus cases as of April-May 2016, coinciding with a decline in ZIKV cases. No patients were identified with ZIKV in our cohort after May 2016. Chikungunya virus was responsible for $42 \%$ of the infections in our control group and coinfected three ZIKV-positive women. Additional infections identified were parvovirus B19 in four ZIKV-negative women and two cases of syphilis, but no adverse pregnancy or infant outcomes were noted in these six patients.

Whether sexual transmission of ZIKV played a role in transmission to pregnant women in our cohort is difficult to assess, since couples usually cohabitate and would presumably have the same type of vector exposure. ZIKV-positive women more frequently had a history of affected family members than ZIKV-negative women.

Links between the current ZIKV epidemic in Brazil and the rise in the number of observed cases of neonatal microcephaly have generated considerable debate about whether the observed phenomenon is real and, if so, whether microcephaly is a direct effect of ZIKV or whether it could be due to potential environmental exposure of pregnant women to teratogenic agents. ${ }^{18}$ There is now a growing body of evidence that suggests a causality link between ZIKV and neonatal structural malformations. ${ }^{19-21}$ Both prenatal and postnatal imaging studies in our cohort showed serious and frequent problems in CNS development in utero, as well as in fetal development overall, with such problems affecting $46 \%$ of 125 
pregnancies and $42 \%$ of 117 live-born infants. Though the clinical illness in women has similarities to rubella, ${ }^{22}$ the effects in the unborn infants differ. With rubella, the time window for adverse outcomes in utero occurs in the first 16 weeks of pregnancy. ${ }^{23-25} \mathrm{In}$ contrast, with ZIKV, the time window appears to be throughout pregnancy. ZIKV pathogenicity was evident in our cohort even in the presence of a "control" group that was affected by chikungunya virus, which is also linked to adverse pregnancy outcomes, particularly fetal loss. ${ }^{26}$

Fetuses infected in the first trimester had findings suggestive of pathologic change during embryogenesis, but CNS abnormalities were seen in fetuses infected as late as 39 weeks of gestation, which underscores the CNS viral tropism. Potential findings suggestive of placental insufficiency were identified in infants who were born small for gestational age or who were born after signs of fetal distress. Microcephaly as detected by ultrasonography and confirmed at birth was noted in four cases (3.4\%), but in only one case was it an isolated finding that was not present in conjunction with fetal growth restriction. Nevertheless, two cases of microcephaly were found to be disproportionate. Although microcephaly has been widely discussed in relation to ZIKV infection, it is important to note that other findings such as cerebral calcifications and fetal growth restriction were present more frequently. Many of the clinical and radiographic abnormalities identified in our cohort have been described in prior case series. ${ }^{27,28}$ We have observed a variety of neurologic findings, including visual and hearing deficits, seizure activity, hypertonicity, spasticity, hyperreflexia, contractures, dysphagia, and feeding difficulties. A troubling aspect of our study is that children were assessed in early infancy, when some subtle neurologic manifestations of disease are difficult to identify. On further follow-up, we suspect that additional clinical manifestations of neurologic disease may be identified in infants who were not previously found to have abnormalities.

Our results should be interpreted with caution since they reflect individual neurologic assessments and imaging performed in early infancy and with knowledge of in utero ZIKV infection status. All infants are still being monitored and will be periodically reevaluated with neurodevelopmental assessments. It is important to note that the potential effect of asymptomatic ZIKV infection during pregnancy was not assessed in this cohort.

In summary, our data show that the risk of severe adverse pregnancy and infant outcomes after maternal ZIKV infection was substantial.

\section{Supplementary Material}

Refer to Web version on PubMed Central for supplementary material.

\section{Acknowledgments}

Supported by Departamento de Ciência e Tecnologia (DECIT) do Ministério da Saúde do Brasil and grants from Coordenacao de Aperfeicoamento de Pessoal de Nivel Superior (CAPES/88887.116627/2016-01); the Bill and Melinda Gates Foundation, Grand Challenges Explorations (OPP112887); and the National Institute of Allergy and Infectious Diseases (NIAID) of the National Institutes of Health (AI AI28697).

We thank the women who enrolled in this study; Ms. Carolina Melo and Mr. Cesare Bianco Junior for technical laboratory support; the Fiocruz Zika field team who helped make our work possible; Dr. Cristina Cassetti and Ms. 
Ann Namkung from the NIAID for their assistance and support; Mr. Marcelo dos Santos for assistance with the graphics of one of our figures; Drs. Yvonne Bryson and Jerome Zack for their ongoing support of our study; and Drs. Celina Boga and Eliane Chaves Vianna of Centro de Saúde Escola Germano Sinval Faria-Escola Nacional de Saude Publica (ENSP) at Fundacao Oswaldo Cruz (Fiocruz) for their continued support of our pregnancy cohort study.

\section{References}

1. Campos GS, Bandeira AC, Sardi SI. Zika virus outbreak, Bahia, Brazil. Emerg Infect Dis. 2015; 21:1885-6. [PubMed: 26401719]

2. Zanluca C, Melo VC, Mosimann AL, Santos GI, Santos CN, Luz K. First report of autochthonous transmission of Zika virus in Brazil. Mem Inst Oswaldo Cruz. 2015; 110:569-72. [PubMed: 26061233]

3. Musso D. Zika virus transmission from French Polynesia to Brazil. Emerg Infect Dis. 2015; 21:1887.

4. Oliveira Melo AS, Malinger G, Ximenes R, Szejnfeld PO, Alves Sampaio S, Bispo de Filippis AM. Zika virus intrauterine infection causes fetal brain abnormality and microcephaly: tip of the iceberg? Ultrasound Obstet Gynecol. 2016; 47:6-7. [PubMed: 26731034]

5. Ventura CV, Maia M, Bravo-Filho V, Góis AL, Belfort R Jr. Zika virus in Brazil and macular atrophy in a child with microcephaly. Lancet. 2016; 387:228.

6. Schuler-Faccini L, Ribeiro EM, Feitosa IM, et al. Possible association between Zika virus infection and microcephaly — Brazil, 2015. MMWR Morb Mortal Wkly Rep. 2016; 65:59-62. [PubMed: 26820244]

7. Calvet G, Aguiar RS, Melo AS, et al. Detection and sequencing of Zika virus from amniotic fluid of fetuses with microcephaly in Brazil: a case study. Lancet Infect Dis. 2016; 16:653-60. [PubMed: 26897108]

8. Calvet GA, Filippis AM, Mendonça MC, et al. First detection of autochthonous Zika virus transmission in a HIV-infected patient in Rio de Janeiro, Brazil. J Clin Virol. 2016; 74:1-3. [PubMed: 26615388]

9. Lanciotti RS, Kosoy OL, Laven JJ, et al. Genetic and serologic properties of Zika virus associated with an epidemic, Yap State, Micronesia, 2007. Emerg Infect Dis. 2008; 14:1232-9. [PubMed: 18680646]

10. Santiago GA, Vergne E, Quiles Y, et al. Analytical and clinical performance of the CDC real time RT-PCR assay for detection and typing of dengue virus. PLoS Negl Trop Dis. 2013; 7(7):e2311. [PubMed: 23875046]

11. Ebbing C, Rasmussen S, Kiserud T. Middle cerebral artery blood flow velocities and pulsatility index and the cerebro-placental pulsatility ratio: longitudinal reference ranges and terms for serial measurements. Ultrasound Obstet Gynecol. 2007; 30:287-96. [PubMed: 17721916]

12. Gardosi J, Chang A, Kalyan B, Sahota D, Symonds EM. Customised antenatal growth charts. Lancet. 1992; 339:283-7. [PubMed: 1346292]

13. Tarrant A, Garel C, Germanaud D, et al. Microcephaly: a radiological review. Pediatr Radiol. 2009; 39:772-780. quiz 888-889. [PubMed: 19437006]

14. Villar J, Cheikh Ismail L, Victora CG, et al. International standards for newborn weight, length, and head circumference by gestational age and sex: the Newborn Cross-Sectional Study of the INTERGROWTH-21st Project. Lancet. 2014; 384:857-68. [PubMed: 25209487]

15. Castanha PM, Cordeiro MT, Martelli CM, Souza WV, Marques ET Jr, Braga C. Force of infection of dengue serotypes in a population-based study in the northeast of Brazil. Epidemiol Infect. 2013; 141:1080-8. [PubMed: 22800513]

16. Gourinat AC, O'Connor O, Calvez E, Goarant C, Dupont-Rouzeyrol M. Detection of Zika virus in urine. Emerg Infect Dis. 2015; 21:84-6. [PubMed: 25530324]

17. Epidemiological alert: neurological syndrome, congenital malformations, and Zika virus infection — implications for public health in the Americas. Washington, DC: Pan American Health Organization; Dec 1. 2015

18. Butler D. Zika virus: Brazil's surge in small-headed babies questioned by report. Nature. 2016; 530:13-4. [PubMed: 26842033] 
19. Rasmussen SA, Jamieson DJ, Honein MA, Petersen LR. Zika virus and birth defects - reviewing the evidence for causality. N Engl J Med. 2016; 374:1981-7. [PubMed: 27074377]

20. Mlakar J, Korva M, Tul N, et al. Zika virus associated with microcephaly. N Engl J Med. 2016; 374:951-8. [PubMed: 26862926]

21. Pacheco, O., Beltrán, M., Nelson, CA., et al. N Engl J Med. Zika virus disease in Colombia preliminary report.

22. Cherry, JD. Cutaneous manifestations of infectious diseases. In: Cherry, JD.Harrison, GJ.Kaplan, SL.Steinbach, WY., Hotez, PJ., editors. Feigin and Cherry's textbook of pediatric infectious diseases. 7. Philadelphia: Elsevier-Saunders; 2014. p. 741-68.

23. Naeye RL, Blanc W. Pathogenesis of congenital rubella. JAMA. 1965; 194:1277-83. [PubMed: 5898080]

24. Plotkin SA, Boue A, Boue JG. The in vitro growth of rubella virus in human embryo cells. Am J Epidemiol. 1965; 81:71-85. [PubMed: 14246083]

25. Sever JL, Schiff GM, Huebner RJ. Frequency of rubella antibody among pregnant women and other human and animal populations: a report from the Collaborative Study of Cerebral Palsy. Obstet Gynecol. 1964; 23:153-9. [PubMed: 14117317]

26. Villamil-Gómez W, Alba-Silvera L, Menco-Ramos A, et al. Congenital chikungunya virus infection in Sincelejo, Colombia: a case series. J Trop Pediatr. 2015; 61:386-92. [PubMed: 26246086]

27. Barcellos C, Xavier DR, Pavão AL, et al. Increased hospitalizations for neuropathies as indicators of Zika virus infection, according to Health Information System Data, Brazil. Emerg Infect Dis. 2016; 22:1894-9. [PubMed: 27603576]

28. Soares de Oliveira-Szejnfeld P, Levine D, Melo AS, et al. Congenital brain abnormalities and Zika virus: what the radiologist can expect to see prenatally and post-natally. Radiology. 2016; 281:203-18. [PubMed: 27552432]

\section{APPENDIX}

The authors' full names and academic degrees are as follows: Patrícia Brasil, M.D., Ph.D., José P. Pereira, Jr., M.D., M. Elisabeth Moreira, M.D., Ph.D., Rita M. Ribeiro Nogueira, M.D., Ph.D., Luana Damasceno, Pharm.D., Mayumi Wakimoto, M.D., Ph.D., Renata S. Rabello, D.V.M., Ph.D., Stephanie G. Valderramos, M.D., Ph.D., Umme-Aiman Halai, M.D., Tania S. Salles, M.D., Ph.D., Andrea A. Zin, M.D., Ph.D., Dafne Horovitz, M.D., Ph.D., Pedro Daltro, M.D., Ph.D., Marcia Boechat, M.D., Ph.D., Claudia Raja Gabaglia, M.D., Ph.D., Patrícia Carvalho de Sequeira, Ph.D., José H. Pilotto, M.D., Ph.D., Raquel Medialdea-Carrera, Ph.D., Denise Cotrim da Cunha, M.D., Liege M. Abreu de Carvalho, M.D., Marcos Pone, M.D., André Machado Siqueira, M.D., Ph.D., Guilherme A. Calvet, M.D., Ph.D., Ana E. Rodrigues Baião, M.D., Elizabeth S. Neves, M.D., Ph.D., Paulo R. Nassar de Carvalho, M.D., Renata H. Hasue, Ph.D., Peter B. Marschik, Ph.D., Christa Einspieler, Ph.D., Carla Janzen, M.D., Ph.D., James D. Cherry, M.D., Ana M. Bispo de Filippis, Ph.D., and Karin Nielsen-Saines, M.D.

The authors' affiliations are as follows: Fundação Oswaldo Cruz (P.B., J.P.P., M.E.M., R.M.R.N., L.D., M.W., R.S.R., T.S.S, A.A.Z., D.H., M.B., P.C.S., J.H.P., R.M.-C., D.C.C., L.M.A.C., M.P., A.M.S., G.A.C., A.E.R.B., E.S.N., P.R.N.C., A.M.B.F.); and Clinica de Diagnostico por Imagem (P.D.) - both in Rio de Janeiro; David Geffen UCLA School of Medicine, Los Angeles (S.G.V., U.-A.H., C.J., J.D.C., K.N.-S.), and Biomedical Research Institute of Southern California, Oceanside (C.R.G.) - both in California; Faculty of Medicine, University of São Paulo, São Paulo (R.H.H.); Medical University of Graz, Graz, Austria (P.B.M., C.E.), and Karolinska Institutet, Stockholm (P.B.M.). 


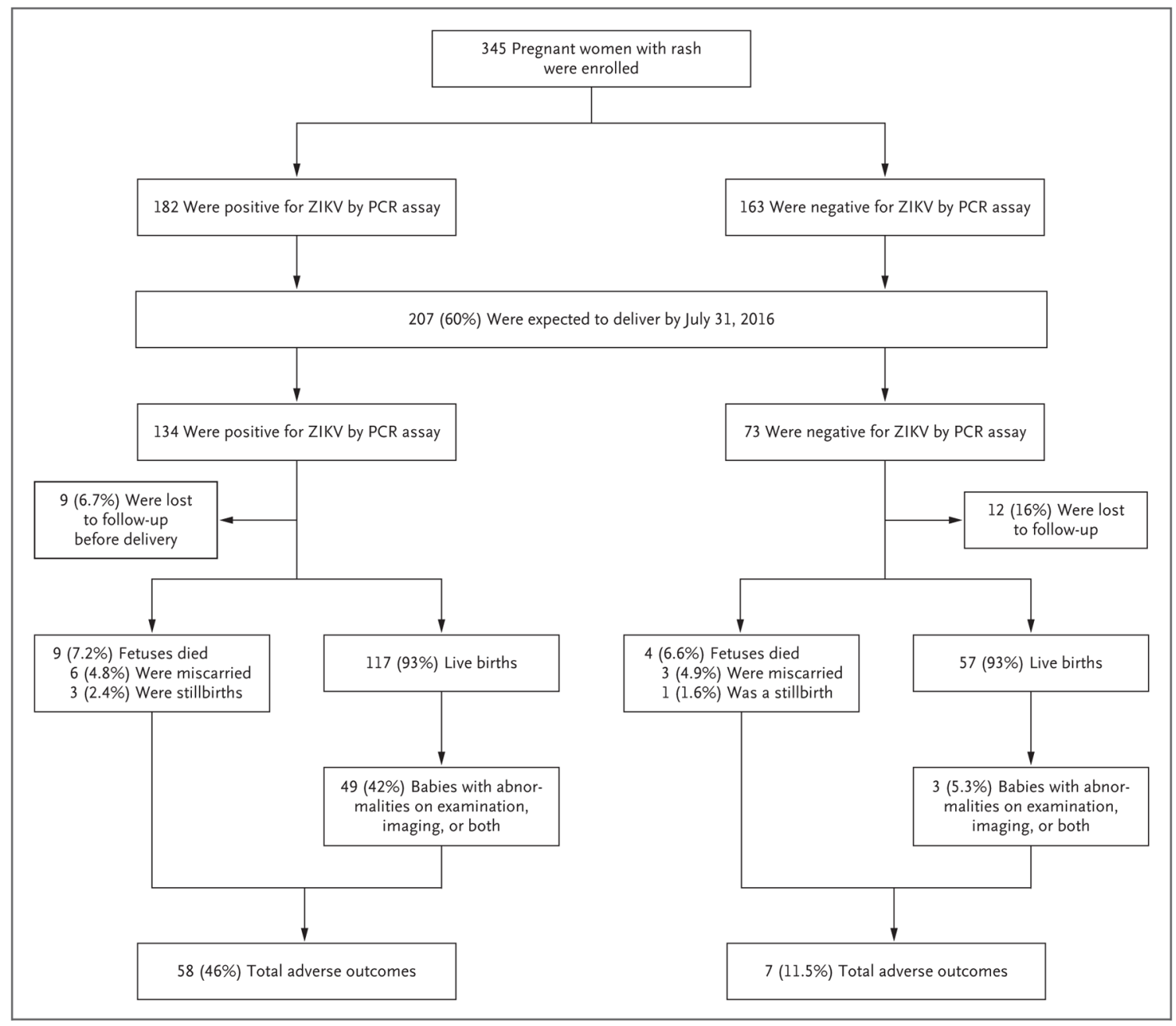

Figure 1. Prospective Maternal Cohort and Pregnancy Outcomes

Among 134 ZIKV-positive women, 9 were lost to follow-up before delivery and 125 had outcomes that could be evaluated; 116 of these pregnancies resulted in 117 live-born babies (there was one set of twins); 9 pregnancies ended in fetal death. One ZIKV-positive mother who had a miscarriage was coinfected with chikungunya virus; two ZIKV-negative mothers whose pregnancies ended in fetal death were infected with chikungunya virus. Three infants of ZIKV-negative mothers were small for gestational age at birth (listed as "abnormalities on examination"); one was born to a mother with confirmed chikungunya virus infection. 


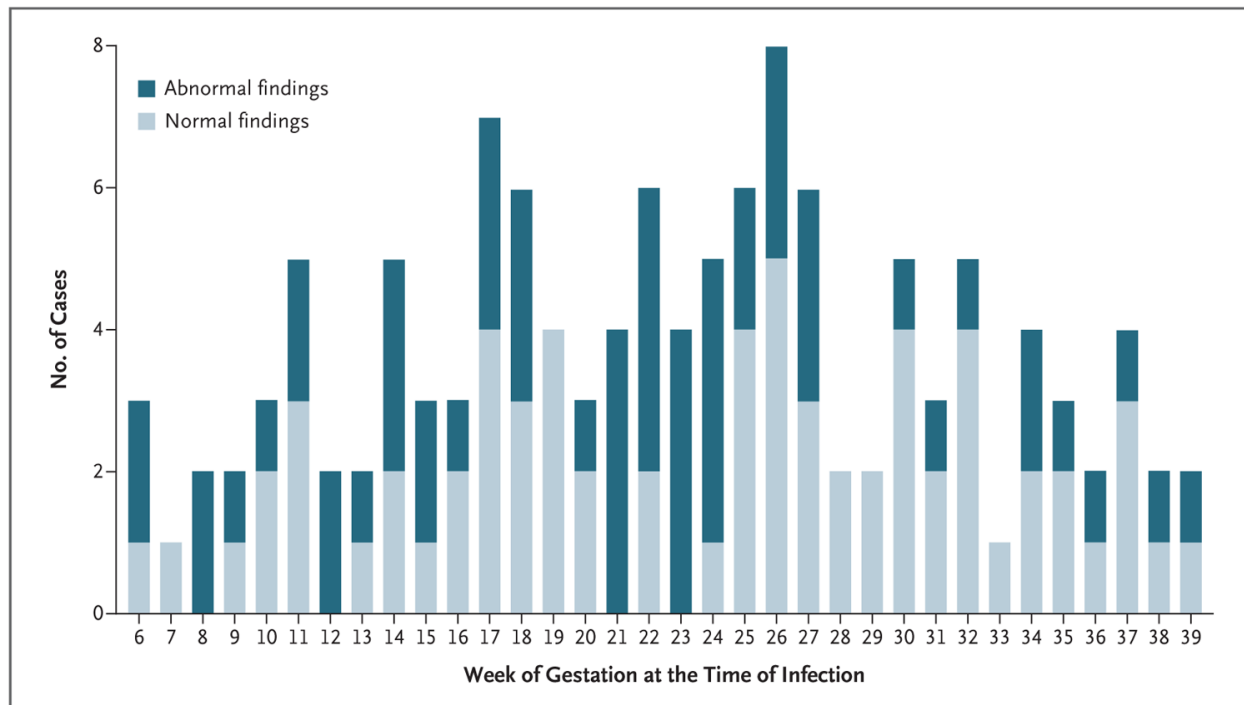

Figure 2. Pregnancy and Infant Outcomes According to the Week of Gestation at the Time of ZIKV Infection

Adverse outcomes included 9 cases of fetal death in 125 pregnancies (7.2\%) and 49 abnormal clinical findings, imaging findings, or both during the newborn period in 117 infants (42\%) born from 116 pregnancies. Adverse outcomes occurred in women who were infected during the period from 6 to 39 weeks of gestation. Abnormalities are detailed in Table S2 in the Supplementary Appendix. 


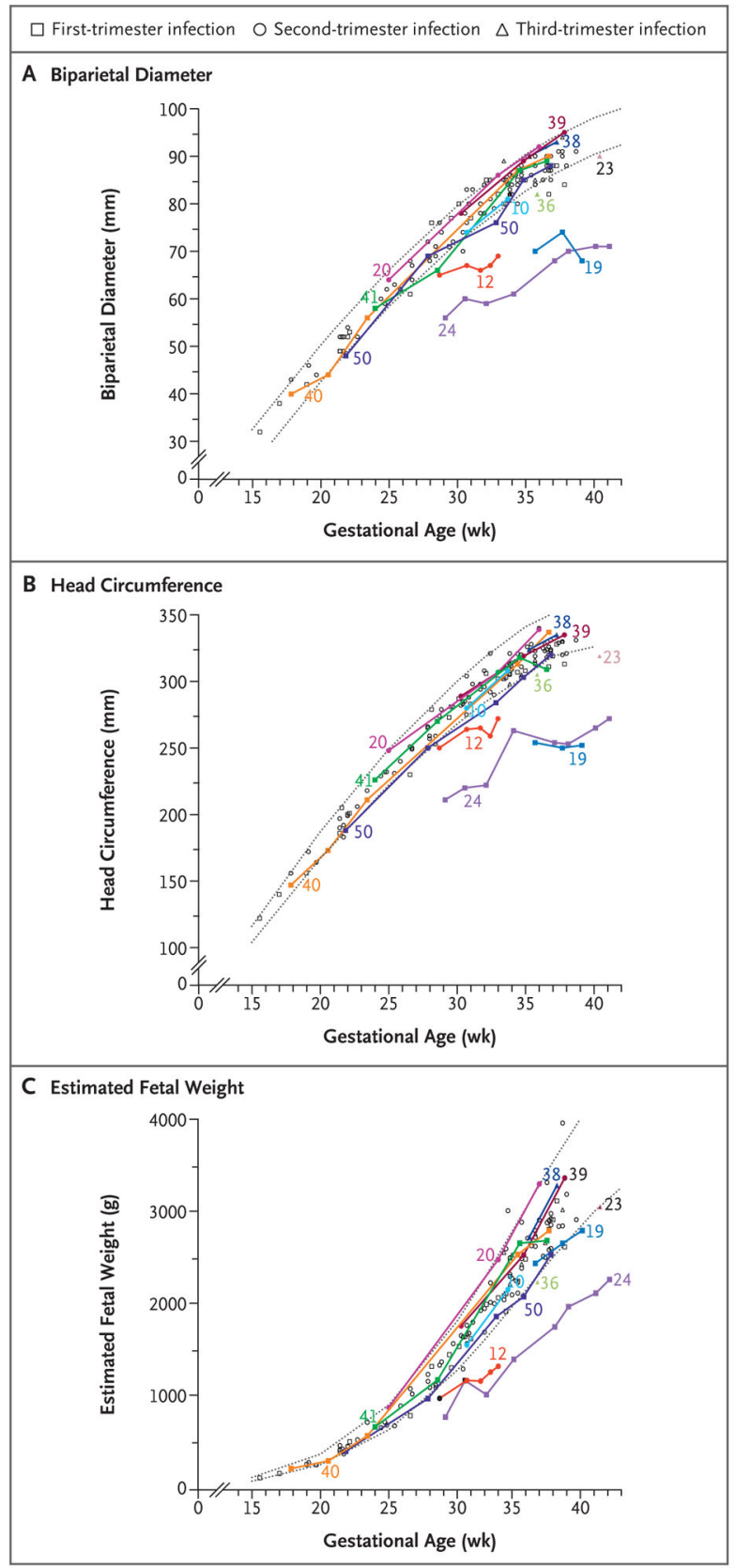

Figure 3. Fetal Biometric Variables as Measured on Ultrasonography

Fetal measurements of biparietal diameter (Panel A), head circumference (Panel B), and estimated fetal weight (Panel C), plotted according to gestational age, are shown for each fetus of ZIKV-positive women who had ultrasonography performed during pregnancy. Dotted lines show the 10th and 90th percentiles for gestational age, based on established nomograms from www.perinatology.com. Fetal weight curves are based on the Hadlock formula, in which a measurement of less than the 10th percentile is considered to indicate fetal growth restriction. Microcephaly was defined as a head circumference of more than 2 standard deviations below the mean expected for gestational age. Symbols denote the 
trimester of PCR-documented infection (first trimester, <14 weeks; second trimester, 14 to 28 weeks; third trimester, $\geq 29$ weeks). Repeat measurements for the same fetus are connected with a solid line to show growth trajectory. Results for fetuses with abnormal findings are denoted in color and labeled with maternal patient number. Not all measurements were obtained for every fetus at each ultrasound examination. 


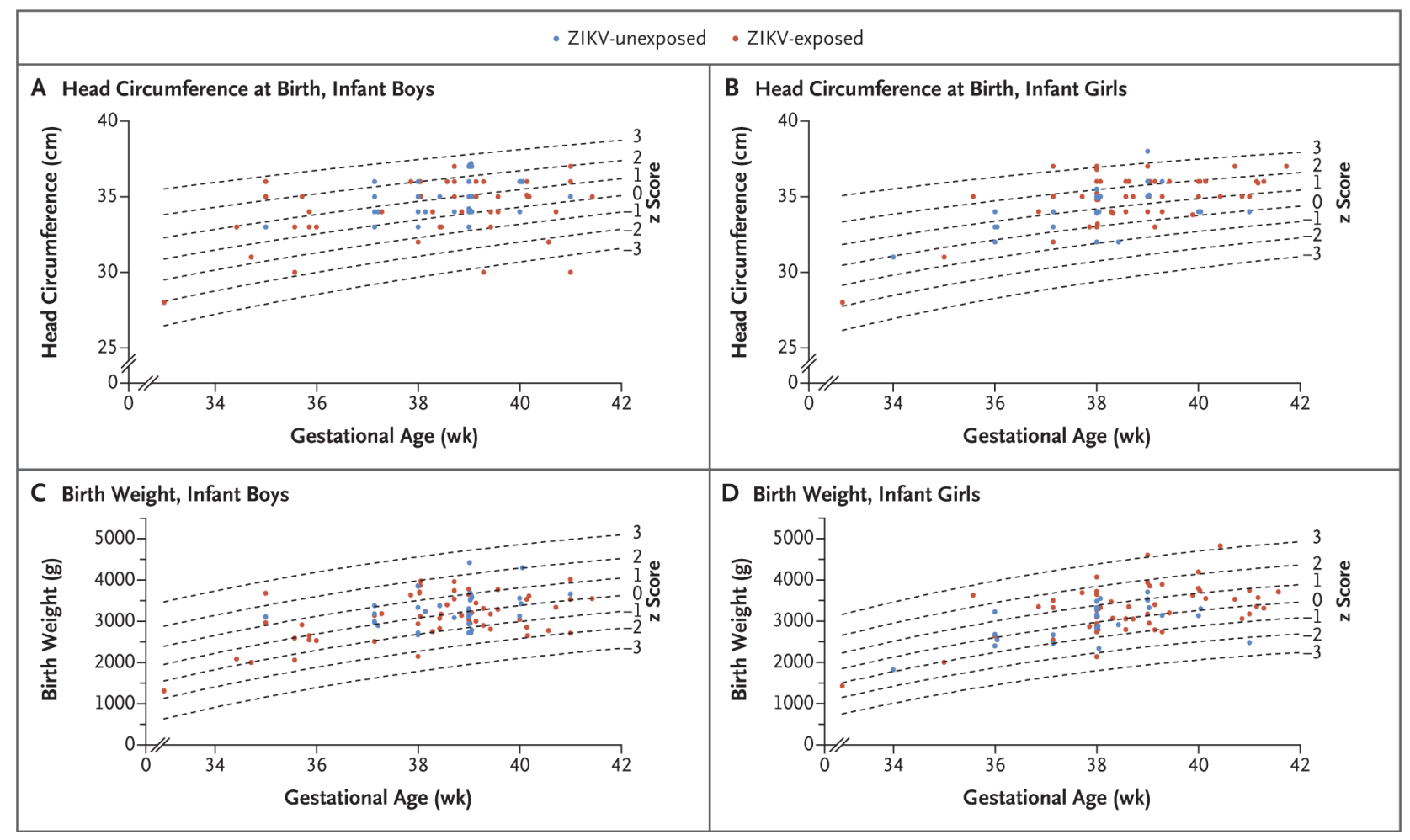

Figure 4. Infant Anthropometric Measures at Birth

Shown are measurements of head circumference at birth in infant boys (Panel A) and girls (Panel B) and estimated birth weight in infant boys (Panel C) and girls (Panel D), according to gestational age at birth. A total of 117 live infants were born to women in our cohort who had positive results for ZIKV on polymerase-chain-reaction (PCR) assays, and 57 were born to women who had negative PCR results for ZIKV. Small for gestational age was defined as a $\mathrm{z}$ score for birth weight of less than -1.28 . Microcephaly was defined as a $\mathrm{z}$ score of less than -2 (moderate) and less than -3 (severe). 


\section{Table 1}

Baseline Demographic and Clinical Characteristics of Women in the Pregnancy Cohort. *

\begin{tabular}{|c|c|c|c|}
\hline Variable & ZIKV-Positive Women (N = 134) & ZIKV-Negative Women (N = 73) & P Value ${ }^{\dagger}$ \\
\hline \multicolumn{4}{|l|}{ Demographics } \\
\hline Age $-\mathrm{yr}$ & & & $0.577^{t}$ \\
\hline Median (IQR) & $31(26-34)$ & $29(25.5-34)$ & \\
\hline Range & $16-46$ & $17-41$ & \\
\hline Other family members ill — no./total no. (\%) & $44 / 115(38.3)$ & $15 / 60(25.0)$ & 0.09 \\
\hline Partner ill — no./total no. (\%) & 14/103 (13.6) & $4 / 53(7.5)$ & 0.30 \\
\hline Use of repellent — no./total no. (\%) & $48 / 80(60.0)$ & $52 / 63(82.5)$ & 0.006 \\
\hline History of dengue - no./total no. $(\%)$ & $33 / 127(26.0)$ & $20 / 69(29.0)$ & 0.74 \\
\hline \multicolumn{4}{|l|}{ Socioeconomic status - no./total no. $(\%)^{\mathcal{E}}$} \\
\hline Income $\mathcal{2} \times$ minimum wage & 48/108 (44.4) & $28 / 63(44.4)$ & 1.0 \\
\hline Income $>2$ to $5 \times$ minimum wage & $38 / 108(35.2)$ & $21 / 63(33.3)$ & 0.87 \\
\hline Income $>5 \times$ minimum wage & 22/108 (20.4) & $14 / 63(22.2)$ & 0.85 \\
\hline \multicolumn{4}{|l|}{ Week of gestation at time of infection } \\
\hline Median (IQR) & $24.5(18-31)$ & $27(23-31)$ & $0.23^{*}$ \\
\hline Range & $5-39$ & $7-36$ & \\
\hline \multicolumn{4}{|l|}{ Distribution - no. $(\%)$} \\
\hline 0 to $\leq 3 \mathrm{wk}$ & $26(19.4)$ & $4(5.5)$ & 0.15 \\
\hline 14 to $\_28 \mathrm{wk}$ & $72(53.7)$ & $41(56.2)$ & 1.0 \\
\hline $229 \mathrm{wk}$ & $36(26.9)$ & $28(38.4)$ & 0.45 \\
\hline \multicolumn{4}{|l|}{ Symptoms - no./total no. (\%) } \\
\hline \multicolumn{4}{|l|}{ Rash $\mathscr{I}$} \\
\hline Any & $134 / 134(100)$ & $73 / 73(100)$ & $0.24 t^{t}$ \\
\hline Median duration (IQR) & $5(4-7)$ & $4(3-6.5)$ & \\
\hline Range & $1-16$ & $1-12$ & \\
\hline Macular & $57 / 134(42.5)$ & $37 / 73(50.7)$ & 0.31 \\
\hline Maculopapular & $57 / 134(42.5)$ & $19 / 73(26.0)$ & 0.02 \\
\hline Other & 20/134 (14.9) & $17 / 73(23.3)$ & 0.18 \\
\hline Pruritus & $116 / 129(89.9)$ & $64 / 73(87.7)$ & 0.64 \\
\hline Arthralgia or arthritis & $81 / 130(62.3)$ & $51 / 73(69.9)$ & 0.29 \\
\hline Conjunctival injection & $73 / 127(57.5)$ & $29 / 72(40.3)$ & 0.03 \\
\hline Headache & 69/127 (54.3) & $47 / 73(64.4)$ & 0.18 \\
\hline Fatigue or malaise & $66 / 127(52.0)$ & $55 / 73(75.3)$ & 0.002 \\
\hline Retro-orbital pain & $53 / 131(40.5)$ & $29 / 73(39.7)$ & 1.0 \\
\hline Myalgia & $53 / 130(40.8)$ & $43 / 69(62.3)$ & 0.005 \\
\hline Lymphadenopathy & $48 / 125(38.4)$ & $19 / 70(27.1)$ & 0.12 \\
\hline
\end{tabular}

N Engl J Med. Author manuscript; available in PMC 2017 June 15. 


\begin{tabular}{|lccc|}
\hline Variable & ZIKV-Positive Women $(\mathbf{N}=\mathbf{1 3 4})$ & ZIKV-Negative Women $(\mathbf{N}=\mathbf{7 3})$ & $\mathbf{P}^{\text {Value }}{ }^{\dagger}$ \\
\hline Localized & $22 / 39(56.4)$ & $5 / 10(50.0)$ & 0.74 \\
\hline Generalized & $17 / 39(43.6)$ & $5 / 10(50.0)$ & 0.74 \\
\hline Paresthesia & $36 / 100(36.0)$ & $26 / 67(38.8)$ & 0.75 \\
\hline Edema & $54 / 99(54.5)$ & $37 / 73(50.7)$ & 0.64 \\
\hline Fever, body temperature $\geq 37.5^{\circ} \mathrm{C}$ & $34 / 124(27.4)$ & $42 / 72(58.3)$ & $<0.001$ \\
\hline Duration $<24 \mathrm{hr}$ & $10 / 20(50.0)$ & $12 / 23(52.2)$ & 1.0 \\
\hline Duration $\geq 24$ to $<72 \mathrm{hr}$ & $10 / 20(50.0)$ & $11 / 23(47.8)$ & 1.0 \\
\hline Fever, body temperature $238^{\circ} \mathrm{C}$ & $3 / 12(25.0)$ & $26 / 38(68.4)$ & 0.02 \\
\hline Photophobia & $44 / 131(33.6)$ & $31 / 73(42.5)$ & 0.29 \\
\hline Anorexia & $37 / 131(28.2)$ & $32 / 73(43.8)$ & 0.03 \\
\hline Diarrhea & $37 / 130(28.5)$ & $23 / 73(31.5)$ & 0.75 \\
\hline Nausea or vomiting & $41 / 131(31.3)$ & $32 / 72(44.4)$ & 0.07 \\
\hline Bleeding, petechia, or enanthema & $12 / 131(9.2)$ & $5 / 73(6.8)$ & 0.79 \\
\hline Abdominal pain & $25 / 131(19.1)$ & $21 / 72(29.2)$ & 0.12 \\
\hline Dizziness or lightheadedness & $28 / 131(21.4)$ & $13 / 67(19.4)$ & 0.85 \\
\hline Respiratory symptoms: coryza, cough, or sore throat & $21 / 123(17.1)$ & $26 / 69(37.7)$ & 0.003 \\
\hline Dysuria & $3 / 119(2.5)$ & $8 / 70(11.4)$ & 0.02 \\
\hline
\end{tabular}

* Differences in denominators across variables are due to missing data. Details on duration of fever or whether lymphadenopathy was generalized or localized were not available for all patients with those findings. IQR denotes interquartile range, and ZIKV Zika virus.

${ }^{\dagger} \mathrm{P}$ values were calculated with Fisher's exact test (two-sided), except as otherwise noted.

The $\mathrm{P}$ value was calculated with the use of an independent-samples median test.

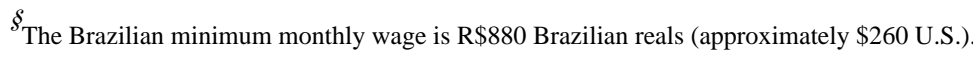

II Rash was an inclusion criterion. 


\section{Table 2}

Pregnancy Outcomes According to ZIKV Exposure.

\begin{tabular}{|c|c|c|c|}
\hline \multirow[t]{2}{*}{ Variable } & $\begin{array}{c}\text { ZIKV-Positive Women }(\mathrm{N}= \\
\text { 134) }\end{array}$ & $\begin{array}{c}\text { ZIKV-Negative Women }(\mathbf{N}= \\
73)\end{array}$ & P Value \\
\hline & \multicolumn{2}{|c|}{ no. of women/total no. (\%) } & \\
\hline Lost to follow-up before birth of infant & $9 / 134(6.7)$ & $12 / 73(16.4)$ & 0.003 \\
\hline Known pregnancy outcomes & 125/134 (93.3) & $61 / 73(83.6)$ & 0.03 \\
\hline Live births, including one set of twins & $116 / 125(92.8)$ & $57 / 61(93.4)$ & 1.00 \\
\hline Adverse pregnancy outcomes $*$ & $58 / 125(46.4)$ & $7 / 61(11.5)$ & $<0.001$ \\
\hline Fetal loss & $9 / 125(7.2)$ & $4 / 61(6.6)$ & 1.00 \\
\hline During first trimester & $5 / 125(4.0)$ & $3 / 61(4.9)$ & 0.72 \\
\hline Rate per total no. of first-trimester maternal infections & $5 / 20(25)$ & $3 / 4(75)$ & 0.72 \\
\hline During second trimester & 2/125 (1.6) & $0 / 61$ & 1.00 \\
\hline Rate per total no. of second-trimester maternal infections & $2 / 71(2.8)$ & $0 / 35$ & 1.00 \\
\hline During third trimester & 2/125 (1.6) & 1/61 (1.6) & 1.00 \\
\hline Rate per total no. of third-trimester maternal infections & $2 / 34(5.9)$ & $1 / 22(4.5)$ & 1.00 \\
\hline \multicolumn{4}{|l|}{ Adverse pregnancy outcomes including fetal loss } \\
\hline First trimester & $11 / 20(55.0)$ & $3 / 4(75.0)$ & $<0.001$ \\
\hline Second trimester & $37 / 72(51.4)^{\dagger}$ & $2 / 35(5.7)$ & $<0.001$ \\
\hline Third trimester & $10 / 34(29.4)$ & $2 / 22(9.1)$ & $<0.001$ \\
\hline Obstetrical complications ${ }^{*}$ & $42 / 107(39.3)$ & $20 / 57(35.1)$ & 0.62 \\
\hline Vaginal deliveries & 20/108 (18.5) & $13 / 57(22.8)$ & 0.54 \\
\hline Cesarean section deliveries & $89 / 108(82.4)$ & $44 / 57(77.2)$ & 0.54 \\
\hline Emergency cesarean section deliveries $\mathcal{\xi}$ & $19 / 81(23.5)$ & $1 / 40(2.5)$ & 0.003 \\
\hline \multicolumn{4}{|l|}{ Positive dengue results } \\
\hline IgG testing & 107/121 (88.4) & $60 / 70(85.7)$ & 0.13 \\
\hline PCR assay & $0 / 134$ & $0 / 73$ & \\
\hline Positive chikungunya IgM or PCR results & $3 / 106(2.8)$ & $25 / 60(41.7)$ & $<0.001$ \\
\hline
\end{tabular}

NEngl J Med. Author manuscript; available in PMC 2017 June 15. 
$t^{t}$ Obstetrical complications included arterial hypertension, eclampsia or preeclampsia, gestational diabetes, acute fetal distress, umbilical prolapse, and abruptio placentae.

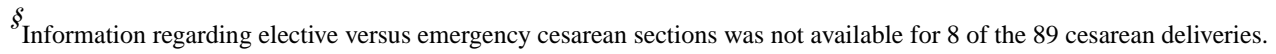




\section{Table 3}

Infant Outcomes According to ZIKV Exposure.

\begin{tabular}{|c|c|c|c|}
\hline Variable & $\begin{array}{l}\text { ZIKV-Exposed Live-Born } \\
\text { Infants }(\mathrm{N}=117)^{*}\end{array}$ & $\begin{array}{l}\text { ZIKV-Unexposed Live-Born } \\
\text { Infants }(\mathbf{N}=\mathbf{5 7})\end{array}$ & P Value \\
\hline Male sex - no. $(\%)$ & $59(50.4)$ & $32(56.1)$ & 0.52 \\
\hline Birth weight $-\mathrm{g}$ & & & 0.79 \\
\hline Median (IQR) & $3192.5(2872.5-3673.5)$ & $3165.0(2890.5-3452.5)$ & \\
\hline Range & $1310-4825$ & $1825-4424$ & \\
\hline Birth length $-\mathrm{cm}$ & & & 0.01 \\
\hline Median (IQR) & $49(47-50)$ & $48(47-49)$ & \\
\hline Range & $35-57$ & $36-52$ & \\
\hline Head circumference $-\mathrm{cm}$ & & & 0.45 \\
\hline Median (IQR) & $35(34-36)$ & $34(34-36)$ & \\
\hline Range & $28-37$ & $31-38$ & \\
\hline \multicolumn{4}{|l|}{ Apgar score } \\
\hline At $1 \mathrm{~min}$ & & & 0.14 \\
\hline Median & 9 & 9 & \\
\hline Range & $1-10$ & $6-10$ & \\
\hline At $5 \mathrm{~min}$ & & & 0.59 \\
\hline Median & 9 & 9 & \\
\hline Range & $5-10$ & $9-10$ & \\
\hline $\begin{array}{l}\text { Admitted to the NICU immediately after birth }- \text { no./total } \\
\text { no. }(\%)\end{array}$ & $22 / 107(20.6)$ & $3 / 55(5.5)$ & 0.01 \\
\hline Duration of NICU stay — days & & & 1.0 \\
\hline Median (IQR) & $5(2-10)$ & $5^{\dagger}$ & \\
\hline Range & $2-30$ & $2-8$ & \\
\hline \multicolumn{4}{|l|}{ Premature - no. $(\%)$} \\
\hline Birth at $<37$ wk of gestation & $17(14.5)$ & $6(10.5)$ & 0.63 \\
\hline Birth at $<35$ wk of gestation & $5(4.3)$ & $1(1.8)$ & 0.66 \\
\hline Small for gestational age - no./total no. (\%) & $10 / 116(8.6)$ & $3 / 57(5.3)$ & 0.06 \\
\hline Microcephaly - no. $(\%)^{*}$ & $4(3.4)$ & 0 & 0.31 \\
\hline Proportionate microcephaly & $2(1.7)$ & 0 & 1.00 \\
\hline
\end{tabular}

N Engl J Med. Author manuscript; available in PMC 2017 June 15. 


\begin{tabular}{|lccc|}
\hline Variable & $\begin{array}{c}\text { ZIKV-Exposed Live-Born } \\
\text { Infants (N = 117) }\end{array}$ & $\begin{array}{c}\text { ZIKV-Unexposed Live-Born } \\
\text { Infants (N = 57) }\end{array}$ & P Value \\
\hline Disproportionate microcephaly & $2(1.7)$ & 0 & 1.00 \\
\hline Total number of adverse infant outcomes - no. $(\%)$ & $49(41.9)$ & $3(5.3)$ & $<0.001$ \\
\hline
\end{tabular}

Included is one set of twins.

${ }^{\dagger}$ The IQR was not calculated (NC) since only 3 non-ZIKV-exposed infants were admitted to the NICU.

Froportionate microcephaly is defined as a condition in which the head size is small but is proportional to the weight and length of the infant; disproportionate microcephaly is a condition in which the head size is small relative to the weight and length of the infant. 